\title{
Rod-Scale Design Strategies for Immune-Targeted Delivery System toward Cancer Immunotherapy
}

Xiupeng Wang, ${ }^{*}+$ Shu Ihara, ${ }^{+}$Xia Li, ${ }^{*}{ }^{\dagger}$ Atsuo Ito, ${ }^{\dagger}$ Yu Sogo, ${ }^{\dagger}$ Yohei Watanabe, ${ }^{\S}$ Atsushi Yamazaki,,$*$ Noriko M. Tsuji,,$^{\S}$ Tadao Ohno"

Table. S1 Synthesis $\mathrm{pH}$ value and final size of HA1-5 rods.

\begin{tabular}{|c|c|c|c|}
\hline Sample & pH & Length & width \\
\hline HA1 & 11.22 & $100 \mathrm{~nm}$ & $20 \mathrm{~nm}$ \\
\hline HA2 & 10.90 & $200 \mathrm{~nm}$ & $40 \mathrm{~nm}$ \\
\hline HA3 & 5.63 & $500 \mathrm{~nm}$ & $60 \mathrm{~nm}$ \\
\hline HA4 & 5.61 & $1 \mu \mathrm{m}$ & $80 \mathrm{~nm}$ \\
\hline HA5 & 5.40 & $10 \mu \mathrm{m}$ & $200 \mathrm{~nm}$ \\
\hline
\end{tabular}

Table S2. PH value of cell culture medium without and with the addition of HA1 5 rods.

\begin{tabular}{|l|l|}
\hline medium & RT. pH value. \\
\hline RPMI1640+10\%FBS. & $8.17 \pm 0.2$ \\
\hline HA1+RPMI1640+10\%FBS. & $8.23 \pm 0.2$ \\
\hline HA2+RPMI1640+10\%FBS. & $8.24 \pm 0.3$ \\
\hline HA3+RPMI1640+10\%FBS. & $8.23 \pm 0.3$ \\
\hline HA4+RPMI1640+10\%FBS. & $8.25 \pm 0.3$ \\
\hline HA5+RPMI1640+10\%FBS. & $8.24 \pm 0.3$ \\
\hline
\end{tabular}



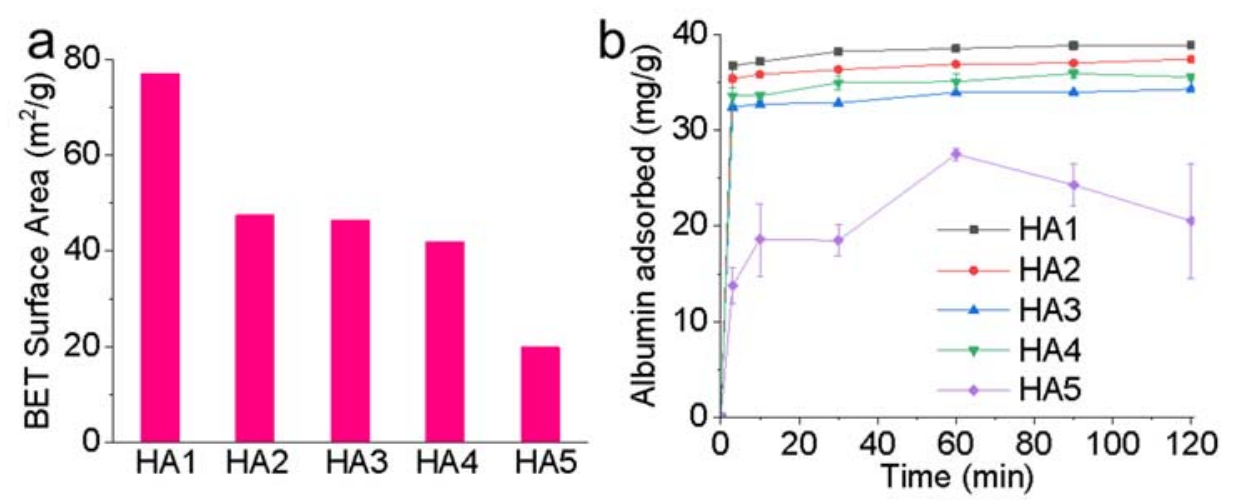

Figure. S1 (a) BET specific surface area of HA1-5 rods. (b) Albumin adsorption by HA1-5 rods in vitro.
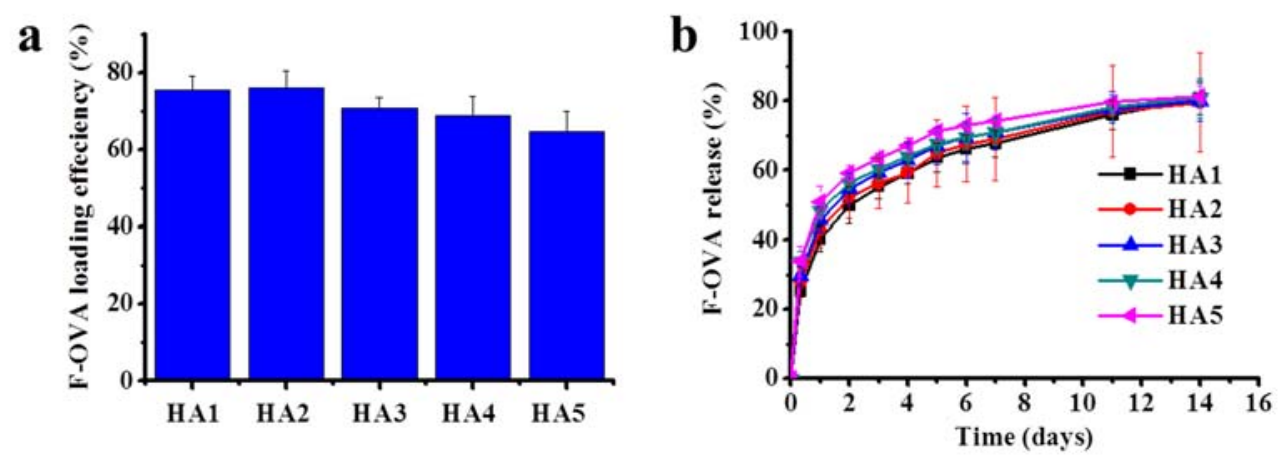

Figure. S2 Model cancer antigen F-OVA adsorption by HA1-5 rods and their release behaviors in $\mathrm{pH}=7.4$ Tris- $\mathrm{HCl}$ buffer supplemented with $10 \%$ serum $(\mathrm{n}=3)$. 

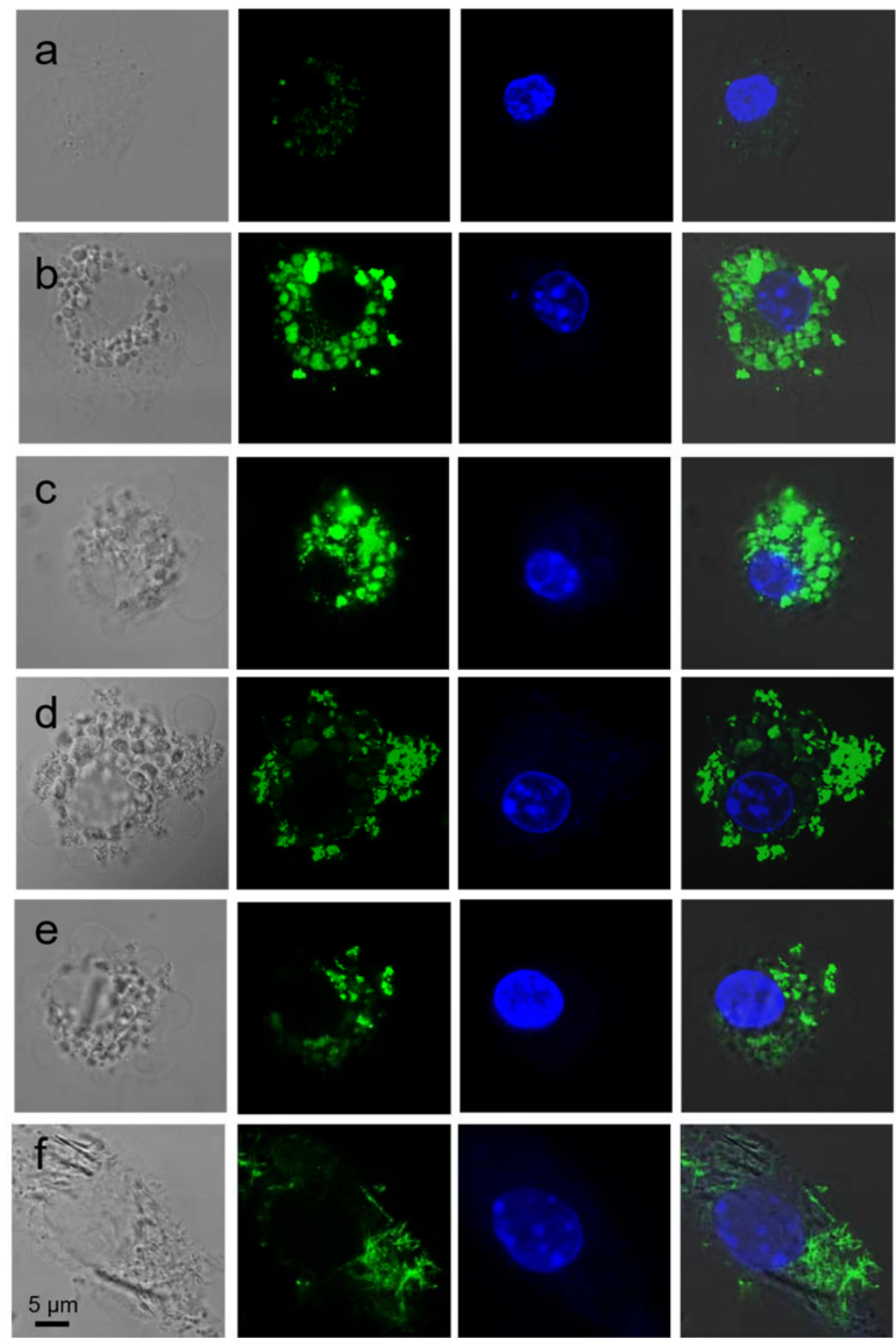

Figure. S3 Representative confocal laser scanning microscope images of free F-OVA (a), HA1-F-OVA (b),

HA2-F-OVA (c), HA3-F-OVA (d), HA4-F-OVA (e) and HA5-F-OVA (f) after $4 \mathrm{~h}$ of culture with BMDCs derived from healthy mice. From left to right, bright field, F-OVA, cell nucleus and merged images, respectively. 

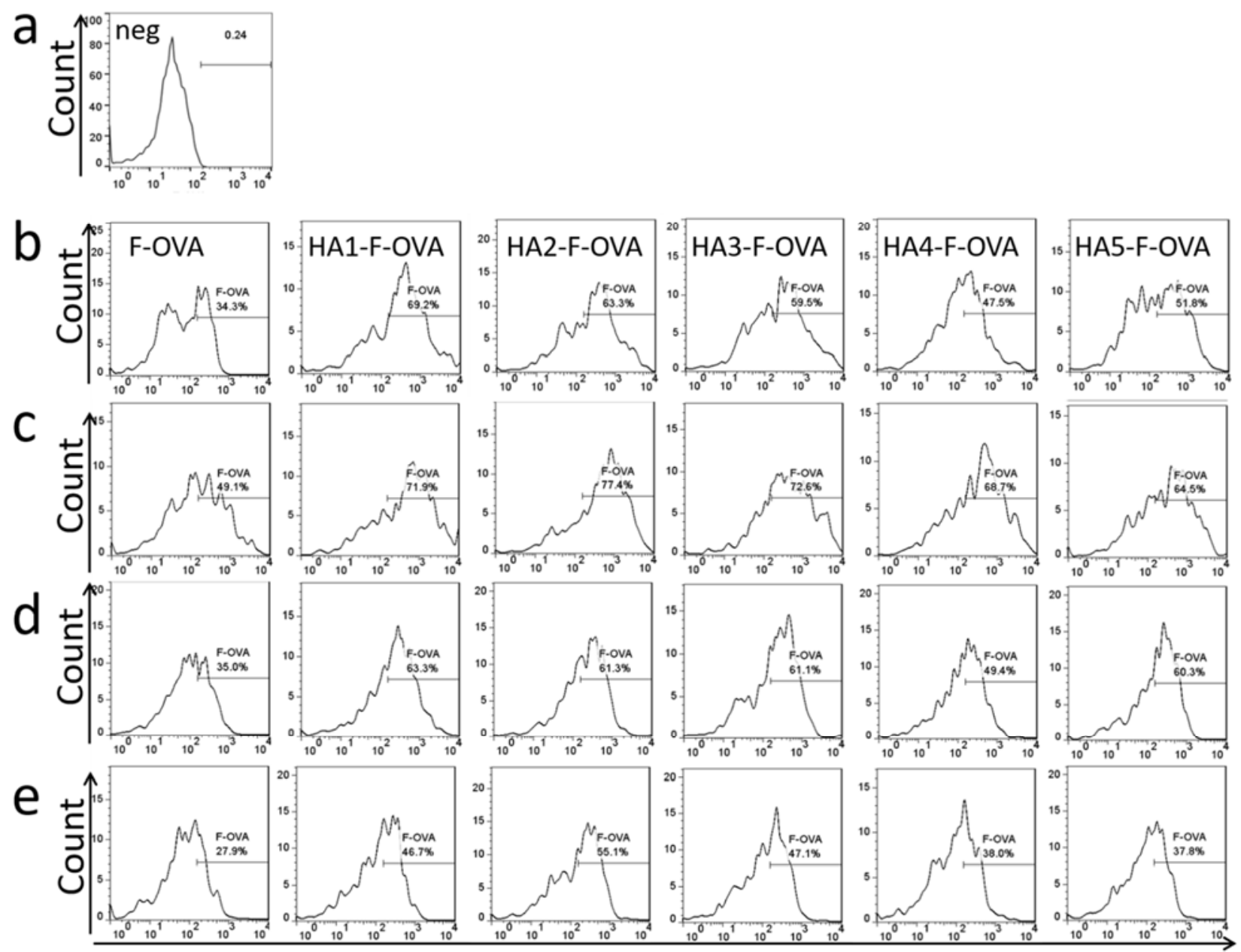

\section{F-OVA}

Figure. S4 BMDCs derived from healthy mice when cocultured with only culture medium (a). Representative cellular uptake of F-OVA by BMDCs derived from healthy mice using flow cytometry after $1 \mathrm{~h}$ (b), $4 \mathrm{~h}$ (c), $12 \mathrm{~h}(\mathrm{~d})$ and $24 \mathrm{~h}(\mathrm{e})$ of coculture with free F-OVA or HA rods loaded with F-OVA. 


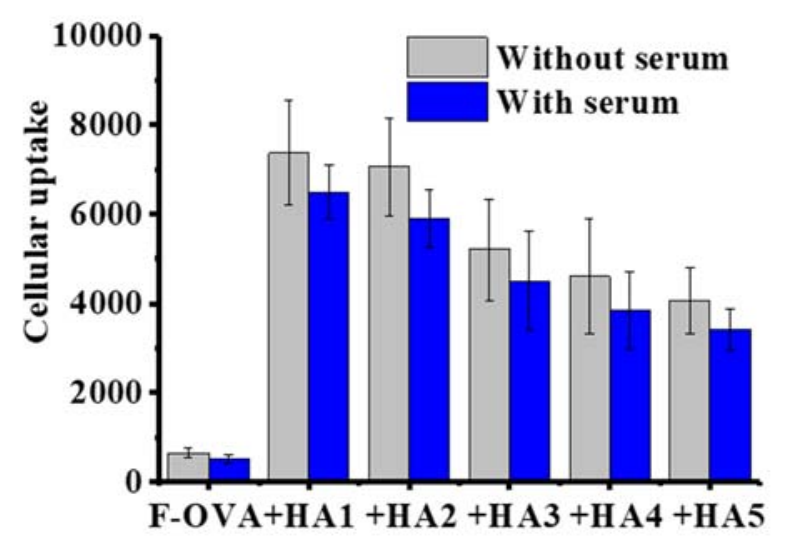

Figure. S5 Quantitative results of F-OVA cellular uptake by BMDCs derived from tumor-bearing mice by fluorescent microplate reader after $4 \mathrm{~h}$ of culture $(n=6, p<0.05)$.

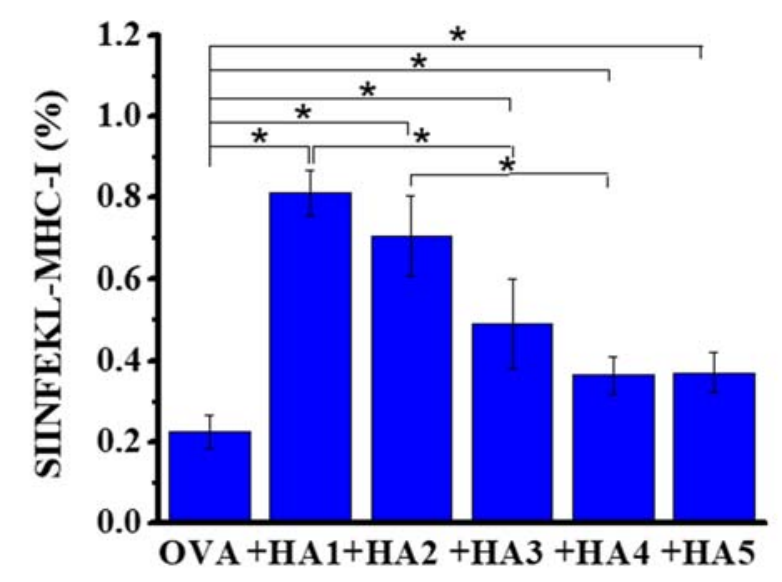

Figure. S6 Cell population of BMDCs derived from tumor-bearing mice presenting SIINFEKL on the MHC class I molecules $(\mathrm{n}=3, \mathrm{p}<0.05)$. 


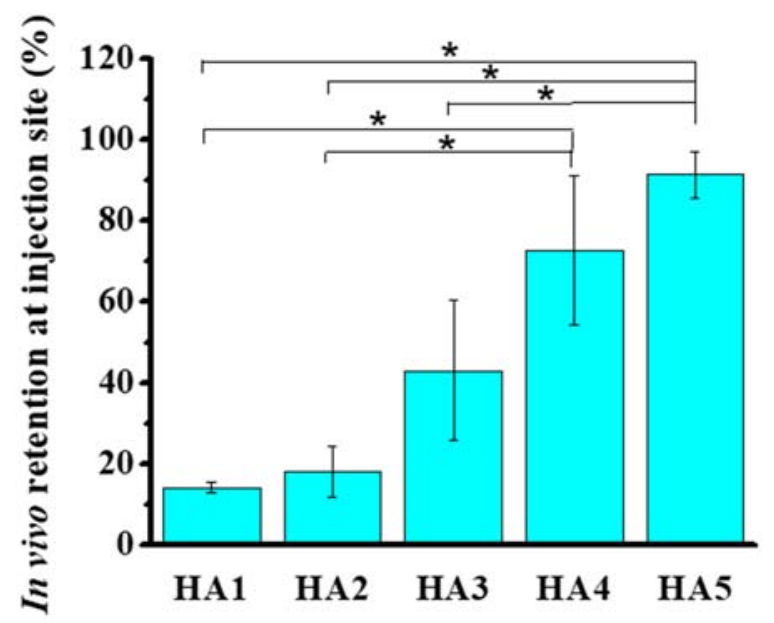

Figure. S7 In vivo HA retention at the injection site after 1 week $(\mathrm{n}=3, \mathrm{p}<0.05)$.
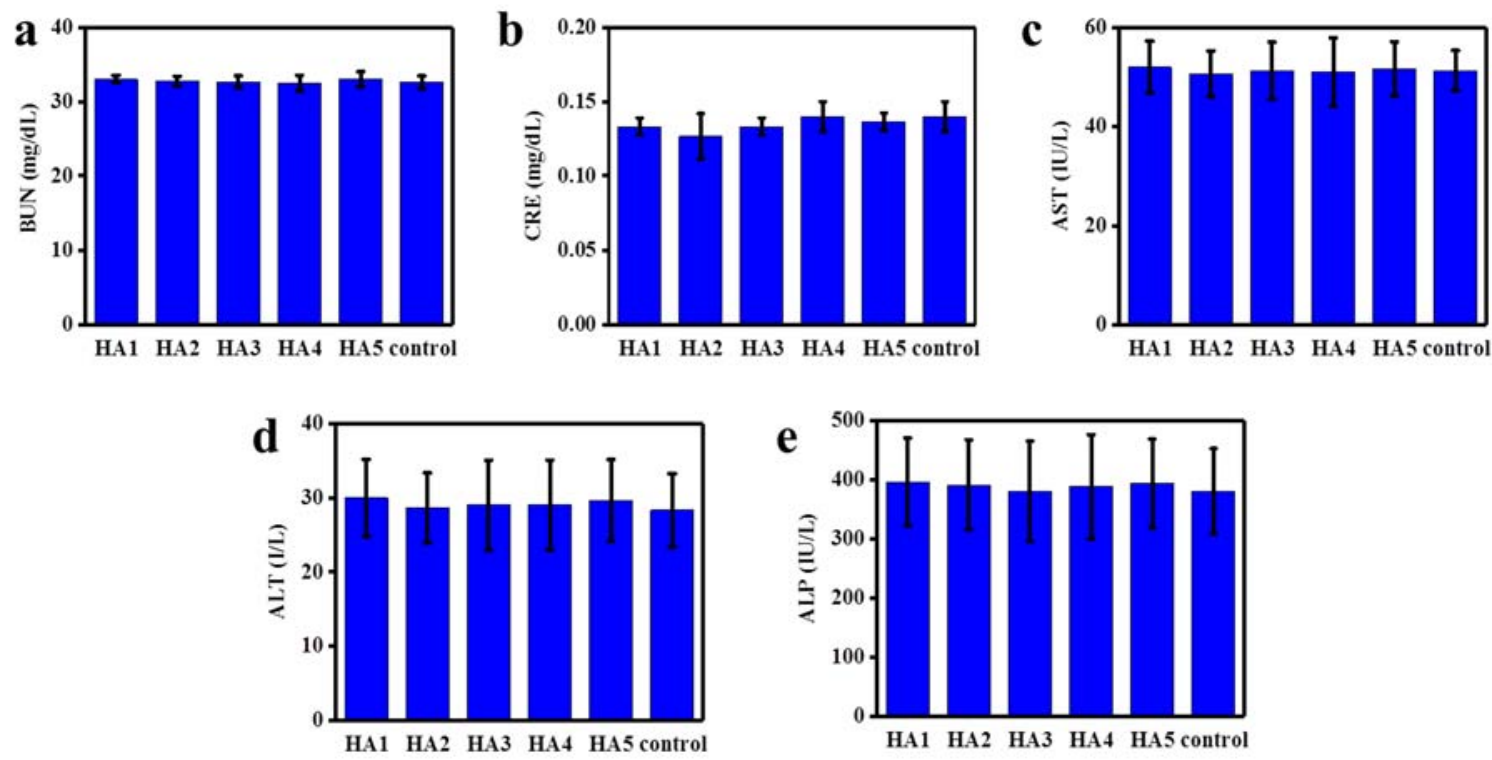

Figure. S8 Biocompatibility of HA rods: Various biochemistry parameters, such as blood urea nitrogen (BUN), creatinine (CRE), aspartate aminotransferase (AST), alanine aminotransferase (ALT), and alkaline phosphatase (ALP) $(n=3, p<0.05)$. 